\title{
THE HEALTH QUEUING GAME
}

\author{
KJETIL K. HAUGEN AND HELGE BREMNES
}

\begin{abstract}
This paper studies agent-to-agent games in competition for a free public resource. The resource is not evidently scarce, scarcity may, however, be the equilibrium outcome. The agents' attributes may differ as well as quality parameters between different public resources. The examples are taken from the health sector. Based on simple two-player simultaneous games of complete information, results regarding agent equilibrium quality choices are derived. Most notably, Nash equilibria of the type: very ill patients choose low quality hospitals (denoted adverse patient allocation in the paper) are demonstrated. Furthermore, it is argued that a situation characterized by patients with relatively mild diseases but large patient variability (big differences between patients regarding the given disease) and a health system with medium competition are prime candidates for Nash equilibria characterized by such Adverse patient allocation effects.
\end{abstract}

\section{INTRODUCTION}

Modern health care industry consumes vast economic resources ${ }^{1}$. Judging modern medical technology and research, it seems hard to predict decreased future resource consumption. Consequently, as a large share of world health care systems are publicly financed, correct regulatory action now and in the future may be of critical importance.

Most of today's health care systems apply a mixture of private and public financing mechanisms. A structural difference may however be observed between countries with "mostly private" (like USA) as opposed to countries with "mostly public" (like Norway or Sweden) financing mechanisms. In countries with a majority of private financing systems, insurance mechanisms dominate while publicly financed systems rely on standard public budgeting mechanisms or (lately) some production oriented mechanisms. As most publicly financed systems supply free services $^{2}$ (publicly provided public goods) and health production-capacity is limited, some rationing mechanism apart from pricing is necessary - typically queuing.

The typical situation - in a country with mostly public health service - may hence be characterized by publicly provided "normal" health production, while relatively small segments are covered by private agents, either for specialized services

$M S C$ (2010): primary 91A05, 91A 80.

Keywords: Health, Queuing, Game Theory, Adverse patient allocation.

${ }^{1}$ In 1991 , US spent $\$ 2.1$ trillion or $13 \%$ of GDP on public and private health care [3]

${ }^{2}$ Some public services apply pricing, but the price is relatively symbolic - no real relation to the true production cost. 
like plastic surgery or for "normal" services and patients with high willingness to pay.

In this paper, we focus on queue formation in a publicly provided (free) health care system. We assume the existence of quality differences between hospitals as well as differences (degree of illness) between patients. We adopt a situation, close to today's situation in Norway ${ }^{3}$, where patients can choose "freely" among different health providers. This freedom of choice introduces difficult decisions for patients and ultimately a gaming situation from the patients' point of view. Any patient would (obviously) like to choose the best hospital with the smallest queue, and conflicts of interest (between patients) will emerge. This situation of conflict is analysed through simple 2 player simultaneous games of complete information.

Section 2 presents a short survey of relevant literature. Section 3 introduces the game models, while the (seemingly) most interesting results are derived in Section 4. The paper ends with some conclusions and suggestions for further work in Section 5.

\section{Literature}

Even though health related research on queues (waiting lists) has become fairly comprehensive, most papers either utilize "standard" general equilibrium approaches or "engineering-oriented" queuing theory. Both these branches of literature are surely important and provide important insight into queuing behaviour. However, fundamental questions regarding agents' equilibrium choices within the theoretical framework of game theory are more sparsely treated. Some noteworthy exceptions do, however, exist. Most of these papers deal with games between regulator/hospital and patient or between hospital/physician and patient. Iversen [6] looks at the game between hospitals and regulators and claims that waiting lists may form a strategic signal for the hospital as long waiting lists may force the regulator to soften the budget constraint. Iversen shows that, given a Stackelberg game formulation (as opposed to a simultaneous game) with the hospital as leader, the equilibrium results in excessive waiting times. In another paper, Iversen [7] treats a somewhat different situation where a "small" private sector may induce equilibrium outcomes with longer waiting lists. Barros et al. [1] and González [5] study "cream skimming" - a situation characterized by public sector treating "hard patients" and private sector "skimming the cream" or treating "easy patients". Barros et al. [1] conclude (perhaps not too surprisingly) that partial "cream skimming" is the typical equilibrium outcome. Farnworth [4] looks at a game between patient and hospital where prices charged to patients are a policy instrument for altering waiting times. He shows that altering prices at some institutions may affect waiting lists at all institutions. Johansen [8] looks at a patient to patient game. He examines, as opposed to our case where player actions

\footnotetext{
${ }^{3}$ Even though we refer to Norway several places in this article, methods and results applies to a general public health service system where queuing is the rationing mechanism and patients are allowed the freedom of hospital choice.
} 
involve choice between hospitals, a one hospital situation ${ }^{4}$ where the players (patients) make timing decisions. His main conclusion involves Nash equilibria in mixed strategies as the typical outcome.

\section{GAME MODELS}

\subsection{The reference model - Equal hospitals and patients and no initial queues}

We assume a simplified setting with 2 hospitals $H_{1}, H_{2}$ and two patients $P_{1}, P_{2}$ where the patients are assumed players in a two-player simultaneous game of complete information. Furthermore, the player actions are assumed to be discrete choices among the two hospitals, and there is no pricing in the system. Queuing is assumed as the (typical) choice of rationing mechanism. Finally, we assume no initial queues and the queue-formation is hence determined by the equilibrium. Pay-offs (or utilities) of the game are assumed equal for all possible decision combinations apart from the only difference due to queuing. That is, $a$ is the (positive) utility for any player given queuing ${ }^{5}$ (patients choose the same hospital) while $b, b>a$ is the utility without queuing.

Given the above assumptions as well as player rationality (utility maximization), the normal form game may be expressed as in Figure 1.

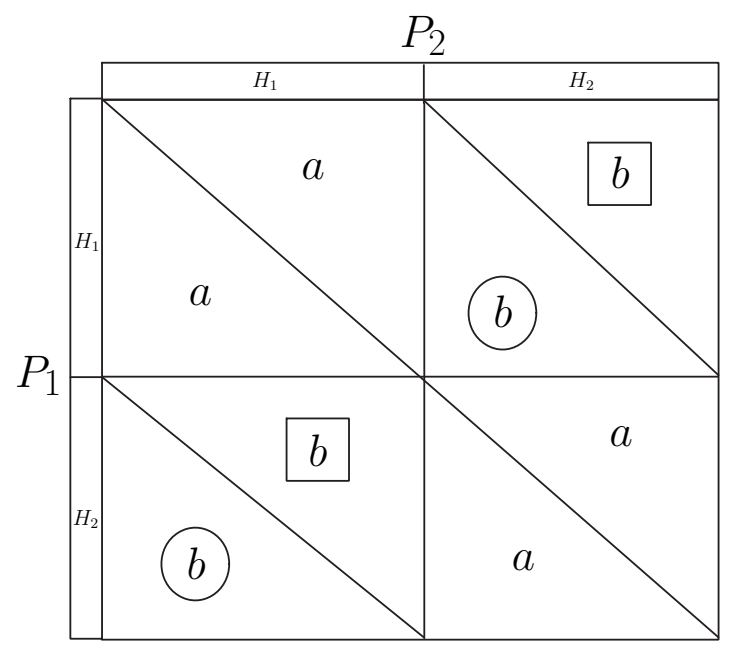

Figure 1. A normal form game - equal patients and hospitals.

\footnotetext{
${ }^{4}$ Strictly speaking, Johansen investigates a general model where the resource not necessarily is a hospital.

${ }^{5}$ Surely, if two patients demand health services at the same (public) hospital with free "production" capacity, only one of the patients will have to wait. As such, this model may perhaps be better explained by assuming a lottery between patients on who will go first. As both patients are assumed "equal", such a lottery should imply equal queuing probability for both, and the positive (expected) utility in this case may for instance be expressed by $b=a-\frac{1}{2} c$. Here, $c$ may be interpreted as the (discounted) cost of queuing.
} 
As Figure 1 indicates, the game is a standard "Chicken" game ${ }^{6}$ with three Nash equilibria, 2 pure (shown by best reply intersections in Figure 1) ${ }^{7}$ and one in mixed strategies (not shown in Figure 1). The practical meaning is straightforward to interpret: minimal congestion, seemingly the politicaly "correct" solution. The intuition is also straightforward, given two equal patients choosing between 2 equal hospitals, with some element of congestion costs; patients ought to end up by avoiding queues.

\subsection{Equal hospitals and unequal patients}

If we assume that one of the patients is very $\mathrm{ill}^{8}$, while the other is not, it seems reasonable to assume that the "very-ill" patient (say $P_{1}$ ) has a utility loss (compared to $P_{2}$ ) by a queue-situation. Consequently, by adding a parameter $\mu$ reflecting this loss, the normal form game of Figure 2 seems to be a reasonable representation of the altered situation.

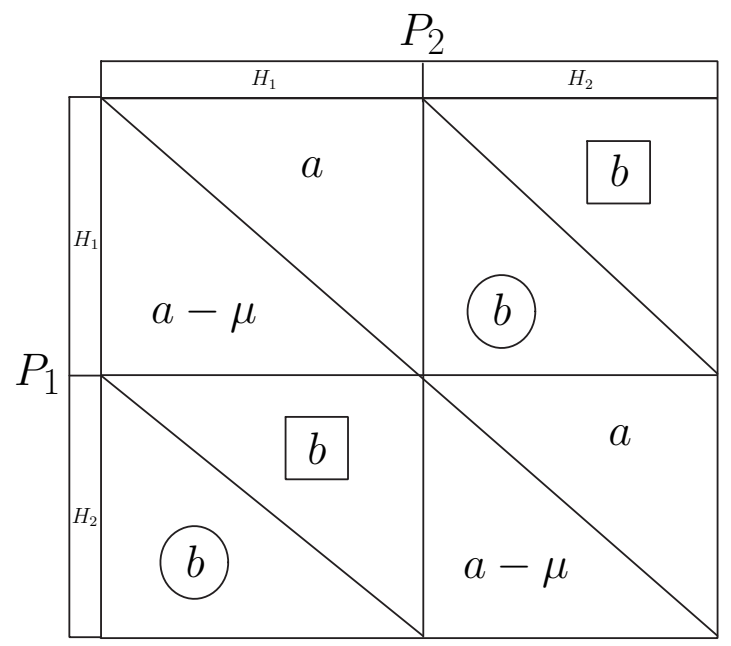

Figure 2. A normal form game - unequal patients and equal hospitals.

As Figure 2 indicates, with the reasonable assumption of $\mu>0$, the Nash equilibria are unchanged. Surely, this is perfectly sensible, as the only difference between figures 1 and 2 is extra congestion costs.

\footnotetext{
${ }^{6}$ The so called "Chicken game" is well treated in Game Theory literature (see for instance [2]) and refers to the game where two hooligans drive against each other on a narrow road, the first to swerve looses faces against his peers, if no one swerves, fatal consequences of a car crash is the "reward" for both players. Strictly speaking, the games which are named "Chicken games" here are not. However, the solution structure (i.e number and type of Nash equilibria) correspond to that of Chicken games. Consequently, we choose to use this naming convention throughout the paper.

${ }^{7}$ Circles denote best reply for patient 1 while squares denote best reply for patient 2 .

${ }^{8}$ Strictly speaking, the model meaning of this term is perhaps better described by "the patient's health deteriorates more while waiting".
} 
To sum up: These simple models show that under an assumption of no quality difference between hospitals and queuing costs, patients choose the hospital with the smallest queue and the outcome is capacity utilization. This is probably the kind of situation that (at least) Norwegian authorities would prefer; equally good hospitals and minimal queue building.

\subsection{Unequal hospitals and equal patients}

This situation will prove to be more interesting. As this case implies variations in hospital quality, a very important distinction will have to be made. Either hospital quality is observed independently of queue length or not. The point is simple. From a patient's point of view, an observed queue (as the only reliable available information on hospital quality) ought to be interpreted as a signal. Rational agents would not queue up - unless they have private information - if alternative hospitals with smaller or non-existing queues exist. Consequently ${ }^{9}$, a long queue should imply better quality than a short one.

For the time being, however, let us assume the opposite situation. That is, hospital quality is observable independently of queue length. This merely means that an observed queue does not imply anything on hospital quality, only disutility of queuing. Given this situation, keeping the congestion cost structure of previous cases $(a, b, b>a)$ and adding a new parameter $\beta>0$ being the positive benefit of choosing $H_{1}$ over $H_{2}{ }^{10}$ yields the game of Figure 3 .

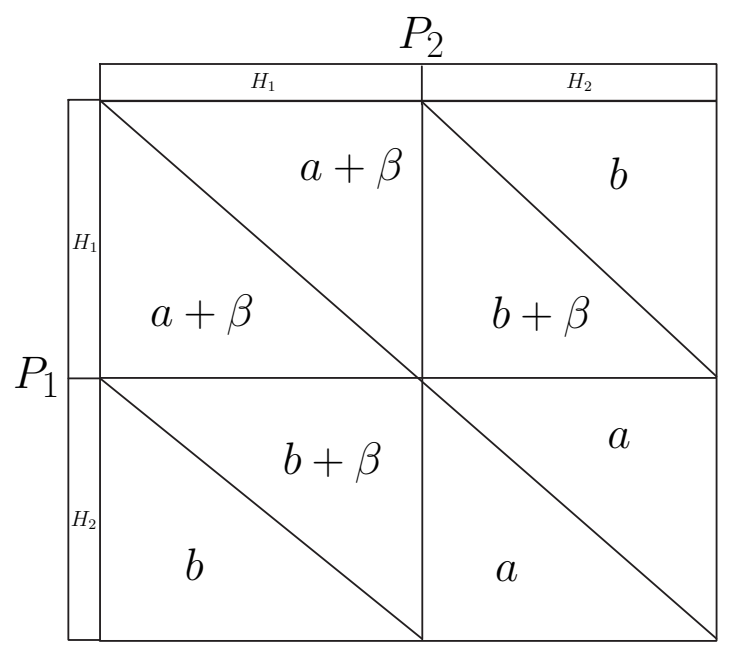

Figure 3. A normal form game - equal patients and unequal hospitals with no utility-signal of queuing.

The game of Figure 3 adds a slightly more complex game theoretic analysis. Two basic situations need to be investigated:

i) $a+\beta<b$,

\footnotetext{
${ }^{9}$ This situation seems to be fairly close to the Norwegian system as of today [9].

${ }^{10}$ This assumption implies (without lack of generality) that hospital 1 is strictly better than hospital 2.
} 
ii) $a+\beta>b$.

\subsubsection{The game of Figure 3 when $a+\beta<b$. If}

$$
a+\beta<b \quad \text { or } \quad a<b-\beta,
$$

then (obviously), given the assumption of $\beta>0$

$$
a<b+\beta
$$

and again the situation imply the "Chicken" game.

3.3.2. The game of Figure 3 when $\boldsymbol{a}+\boldsymbol{\beta}>\boldsymbol{b}$. This situation does, however, imply an interesting change. Let us first interpret the meaning. If $a+\beta>b$ or, equivalently, $\beta>b-a$, the added utility in favour of hospital 1 is larger than the congestion costs. Or more popularly, the above defined situation may be seen as a situation with a large hospital quality variation.

Half of the best replies are determined by the initial assumption of $a+\beta>b$. It is, however, necessary to establish the sign of $(b+\beta)-a$. Assuming

$$
a>b+\beta \Rightarrow \beta<a-b \Rightarrow \beta<0
$$

which clearly violates the assumption of $\beta>0$ and consequently, $a<b+\beta$. Hence, it is straightforward to see that a unique pure Nash equilibrium exists, as shown in Figure 4.

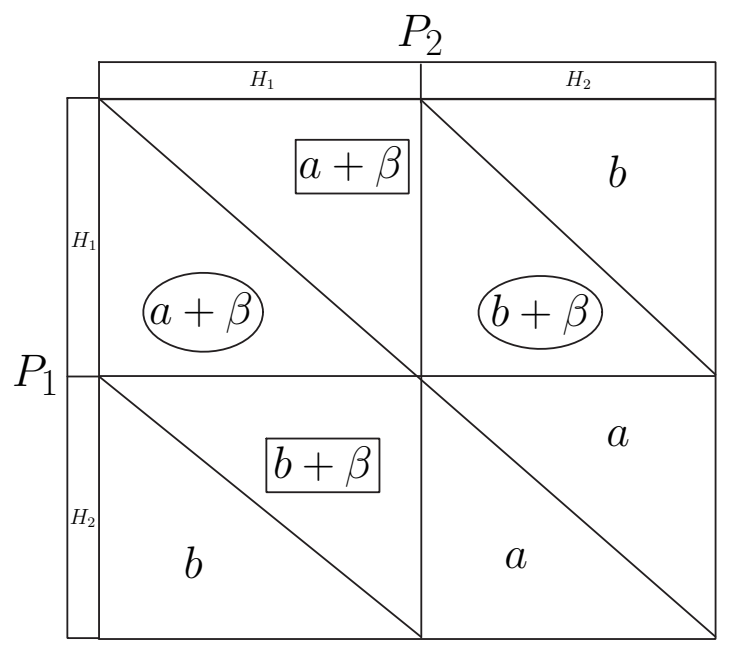

Figure 4. Nash equilibrium in the game of Figure 3.

This situation is intuitive and logical. When the quality difference among hospitals is large enough, patients choose the best hospital with queuing as the consequence. However, this may well be a fairly "unpleasant" solution as seen by a politician. If patients choose only quality hospitals, the remaining (low quality) hospitals will ultimately lack patients. An obvious consequence would be a hospital shut down, seemingly not a very "politically correct" solution - at least not in a sparsely populated and geographically complex country such as Norway. 
As discussed initially in this paragraph, the above modelling reflects a situation where the queue-length does not imply a quality signal. In health systems such as the Norwegian one today, the only quality information available is queue lengths (or waiting lists as Norwegians tend to name them). Any patient observing very different queue lengths among hospitals (with seemingly equal quality) for the same type of treatment, would be very silly if she did not ask the following question: If all hospitals are equally good, how come that most patients choose a subset of hospitals? Obviously, in such a system, a long queue must imply a positive quality signal.

The above description calls for a game of incomplete (and asymmetric) information with sets of "informed" patients and sets of "uninformed" patients. Such an approach is clearly feasible, but judged as out of the scope of this paper ${ }^{11}$. It is however possible to judge consequences of such a situation by a simple approximative technique. If queue-length indicates (positive) hospital quality, it should mean that the "cost of congestion" ought to be smaller given the ii)-situation as opposed to the i)-situation. After all if the length of the queue gives a signal to the patient that the hospital at hand is good, the rational patient would be less reluctant in choosing such a hospital - or be more willing to accept queuing.

Consequently, we can compare the two situations by simply judging the reasoning in subsections 3.3.1 and 3.3.2 given $a$ is closer to $b$. If $b-a$ is decreasing, it is simple to realize that the situation of subsection 3.3.1 becomes less probable compared to the situation of subsection 3.3.2 - that is, a Nash equilibrium where both patients choose one hospital is more likely. Hence, if it is plausible to assume that a public health system that has been run without competitive forces like the Norwegian one, has a large quality variation (typically also with significant asymmetric information between patients) making waiting lists public, may not be a very good idea as the above argumentation indicates a possible outcome of increased congestion.

The point is simple. If the politicians' intention to make waiting lists public is a hope that patients will choose hospitals with small queues, the actual outcome may very well be the opposite. This kind of result ought to be interesting for Norwegian health officials.

\section{Both unequal hospitals and unequal Patients}

As the headline suggests, it is perhaps not too surprising that the final section contains a judgement of the more general situation. Surely, this case contains all the other cases discussed, along with a large set of other situations. We see no point in a full examination of the general case which, in essence, will merely be a complete taxonomy of two-player two-strategy one-shot games.

However, it may prove to be interesting to see if a situation where "very ill" patients are forced (by the game) to choose "very low-quality" hospitals is obtainable. We use the term adverse patient allocation to characterize such outcomes. Surely, such outcomes are definitely not what either patients, hospitals or society would welcome.

\footnotetext{
${ }^{11}$ An obvious candidate for the "category of further work".
} 
In order to judge this question, a more general game model is needed. Actually, "joining" figures 2 and 3 will serve the purpose as shown in Figure 5.

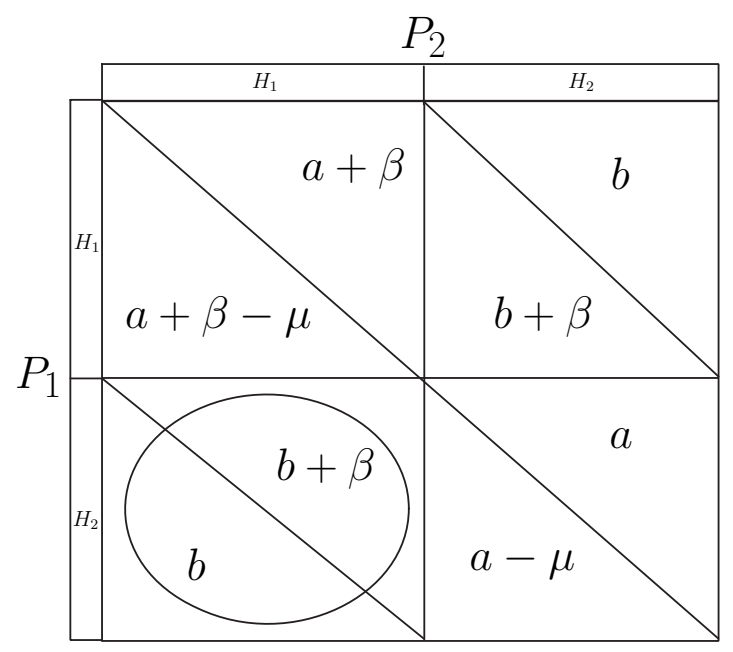

Figure 5. A game with both unequal hospitals and patients.

As indicated by Figure 5, this is a game between a "very ill" patient $\left(P_{1}\right)$ and a less ill patient $\left(P_{2}\right)$ choosing between two hospitals of which $H_{1}$ is better than $H_{2}$. That is, $\beta, \mu>0$. We also keep the assumption of $b>a$.

Given theses assumptions and the case of "very ill" patients choosing "very bad" hospitals, the pure unique Nash equilibrium of $P_{1}$ choosing $H_{2}$ and $P_{2}$ choosing $H_{1}$ is the candidate for examination as indicated by the circle of Figure 5.

In order to secure $\left(H_{2}, H_{1}\right)$ as a pure Nash equilibrium, inequalities (4.1), (4.2) must (at least) be satisfied:

$$
\begin{aligned}
& b>a+\beta-\mu \\
& b+\beta>a
\end{aligned}
$$

Starting with (4.2), we get

$$
b+\beta>a \Rightarrow \beta>a-b .
$$

But, by the initial assumptions $\beta>0, b>a$, the equation (4.3) will always be satisfied.

Examining (4.1) yields (by adding $\beta$ on each side)

$$
b>a+\beta-\mu \Rightarrow b+\beta>a-\mu+2 \beta .
$$

Consequently, as $b+\beta>a-\mu+2 \beta>a-\mu$, we have

$$
b+\beta>a-\mu
$$

or $P_{1}$ 's best reply to $P_{2}$ choosing $H_{2}$ is $H_{1}$. That is, in order to avoid the "Chicken" outcome and secure a pure and unique Nash equilibrium of $\left(H_{2}, H_{1}\right)$-type, it is 
also necessary that equation (4.4) is satisfied

$$
a+\beta>b .
$$

To sum up: both equations (4.4) and (4.1) must be satisfied simultaneously. Rewriting these equations slightly gives

$$
b>a+\beta-\mu \Rightarrow \beta<b-a+\mu
$$

and

$$
a+\beta>b \Rightarrow \beta>b-a
$$

or

$$
b-a<\beta<b-a+\mu .
$$

Inequality (4.5) can be seen as an interval for possible $\beta$-values, i.e., $\beta \in[b-a, b-$ $a+\mu]$. One interesting and obvious conclusion to draw from equation (4.5) is the simple (and somewhat surprising) fact that if we judge the length of the above interval (computed as $(b-a+\mu-(b-a)=\mu$ ) as an indicator on the likelihood

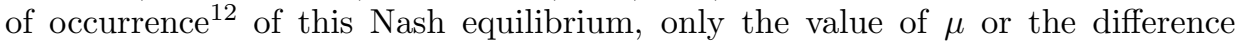
between patients are relevant.

This may be interpreted as follows: In order to minimize the occurrence of this type of equilibrium, a regulator should - for any value of $\beta$ (hospital quality) minimize $\mu$ or try to make patients as equally ill (or fresh) as possible.

The fact that a regulatory objective may involve "picking" patients that are equally ill (or equally fresh) is perhaps not directly intuitive.

Another striking fact of equation (4.5) may be observed by conducting the following argument: Assume that congestion costs $(b-a)$ as well as patient differences $(\mu)$ are given. Avoiding this outcome may be done in two ways, either by making the hospital quality difference sufficiently large or small. Note however, that this type of regulatory action is fairly dangerous. If one fails to make it either small enough or large enough, nothing happens; (either $\beta$ must be made $<b-a$ or $\beta$ must be made $>b-a+\mu$.

\section{CONClusions AND FURTher WORK}

\subsection{Basic findings}

To sum up, there are two basic findings in this paper. First, we have demonstrated (in subsection 3.3) that, given sufficient quality difference between hospitals, a pure unique Nash equilibrium of both patients choosing the "best" hospital is predicted. This may be seen as an explanation of why the introduction of free hospital choice may actually lead to increased waiting lists. The fact that patients prefer quality hospitals if the quality difference is large enough compared to congestion costs is surely neither surprising nor counter intuitive. As such, this finding may seem banal from a game theoretic point of view. However, as the political debate of for instance Norway strongly indicates that making waiting lists public should help patients avoid the queues and hence lead to reduced waiting lists, such a finding might still be of regulatory interest. Actually, it could be argued that making

\footnotetext{
${ }^{12}$ This seems like a reasonable judgement.
} 
waiting lists publicly available may not be the correct regulatory action if the aim is to reduce waiting lists.

Second, and perhaps more important, the findings of Section 4 indicate the existence of Nash equilibria predicting adverse patient allocation. In this context, adverse patient allocation means a situation where the patients with the greatest needs choose the hospitals of lowest quality ${ }^{13}$. Consequently, interpreting equation (4.5) somewhat more might be interesting both from a practical as well as a regulatory point of view.

If we compare health care systems with high and low competition, the central parameter of equation (4.5) is $\beta$, representing the quality differences between hospitals. It is reasonable to assume that high competition will drive hospitals towards equal quality and hence $\beta \rightarrow 0$. For a given positive value of the congestion costs $(b-a)$ the regulatory action is obvious - increase competition. However, as equation (4.5) also indicates, the opposite regulatory action is possible. Given congestion costs, $(b-a)$ and patient differences, $(\mu)$, decreasing competition (i.e. increasing $\beta$ ) so that $\beta>b-a+\mu$ secures the non-satisfaction of equation (4.5). The conclusion, perhaps somewhat counter intuitive, is that health systems with medium competition are the risky ones. If competition is high enough or low enough, equation (4.5) is difficult to satisfy.

An alternative way of viewing this situation is to focus on diagnosis. As $b-a$ represents congestion costs, it is reasonable to state that severe illness such as cancer or heart disease should imply large congestion costs or high $b-a$-values while less severe cases such as rheumatic disease have small $b-a$-values. Consequently, for a given competitive level (given $\beta$ ), the adverse patient allocation mechanism is less likely to occur for severe cases, which is good news. On the other hand, cases with large patient variability (large $\mu$ ) indicate the satisfaction of equation (4.5).

To sum up: A situation characterized by a relatively mild ${ }^{14}$ disease but large patient variability and a health system with medium competition are the typical candidates for satisfaction of equation (4.5). Treatment of rheumatic disease in Norway may perhaps fit such a description fairly well. Note, however, that even severe diseases such as cancer may fit our description of adverse patient allocation if patient variability is large enough and competition is low enough. In such a case, both $\beta$ and $\mu$ are large and perhaps large enough to satisfy (4.5).

\subsection{Model criticism}

The fact that our game-modelling is simplified may of course be criticized . What would happen if the number of players (patients) and strategies (hospitals) increased? After all, the real world contains more than 2 patients and 2 hospitals. Surely, special effects may arise, but it seems safe to conclude that the main structural consequences will remain.

\footnotetext{
${ }^{13}$ Obviously, this situation interpreted as adverse patient allocation implies a reasonable underlying assumption of suboptimal social welfare. It is hard to argue that allocating the most needing patients to the hospitals of lowest quality maximizes social welfare.

14 "Mild" should be interpreted (modelwise) as a disease with relatively low health deterioration due to waiting.
} 
Some might object to the choice of a simultaneous game. After all, patient decisions on hospital choice are not made simultaneously but (perhaps) sequentially. From a practical point of view, such a model criticism may seem reasonable, but still; patients must make choices on where to take treatment without knowing what other patients at that time choose. Hence, our simplified description may not be so bad after all.

The fact that this type of situation is full of asymmetric information is obvious. Patients will not know their opponent players' types, and patient knowledge on hospital quality may differ between patients. To a certain extent, the arguments of subsection 3.3, make up for such considerations, but as stated there, such an approach deserves a paper of its own.

The most significant objection that may be raised against the modelling and results of this paper is perhaps the implicit assumption underlying (Sub)Sections 3.2 and 4 of free hospital choice regardless of disease. The meaning is straightforward. It may well be that a regulator predicts the consequences of free hospital choice as discussed here and chooses to lock special patients to special hospitals. If this is the case, the gaming problems discussed previously are of course less interesting. On the other hand, if a regulator makes such a choice, the concept of free hospital choice becomes misleading, and it may be argued that a regulator bites his own tail. Even if this is a possible practical outcome, it seems obvious that only special (very severe cases) may be embraced by such an arrangement. As such, our modelling may still be valid but within a subset of diagnostic cases.

\subsection{Generality of results}

Finally, it is clear that the modelling and results of this paper are not restricted to health services only. The generality of modelling, models and results are surely applicable to a wider set of public services - typically categorized as public goods in economic theory. Education and free legal services may serve as typical examples. In practice, some differences may of course need remodelling - public education implies a re-queuing mechanism for instance. But, given an educational system without re-queuing, our case would transform nicely to the existence of Nash equilibria where the good students end up in the bad universities. Something

perhaps fairly familiar for those acquainted with the Scandinavian educational systems.

\section{REFERENCES}

[1] P. P. Barros and P. Olivella, Waiting lists and patient selection, J. Econ. Manage. Strat. 14 (2005), 513-527.

[2] K. Binmore, Fun and Games, D. C. Heath and Company, Lexington, Massachusettes, Toronto, 1992.

[3] M. Carter, Diagnosis: Mismanagement of Resources, OR/MS Today, 2002.

[4] M. G. Farnworth, A game theoretic model of the relationship between prices and waiting times, J. Health Econ. 22 (2003), 47-60.

[5] P. González, On policy implications of transferring patients to private practice, Health Econ. 14 (2005), 513-527.

[6] T. Iversen, A theory of hospital waiting lists, J. Health Econ. 12 (1993), 55-71.

[7] T. Iversen, The effect of a private sector on the waiting time in a National Health Service, J. Health Econ. 16 (1997), 381-396. 
[8] L. Johansen, Queues (and rent-seeking) as non-cooperative games, emphasizing mixed strategy solutions, in: F. Førsund (ed.), Collected Works of Leif Johansen, vol. 2, North-Holland, Amsterdam, 1987, 827-876.

[9] R. Riise and A. Lutro, VG-tabbe om sykehustall (in Norwegian), downloaded from: http://pub.tv2.no/nettavisen/innenriks/article490089.ece, Nettavisen, Norway, November 2005.

Kjetil K. Haugen, Faculty of Economics, Informatics and Social Science, Molde University College, Box 2110, 6402 Molde, Norway

e-mail: kjetil.haugen@himolde.no

Helge Bremnes, More Research Molde AS, Britvegen 4, N-6411, Molde, Norway e-mail: helge.bremnes@himolde.no 